General Relativity and Gravitation manuscript No.

(will be inserted by the editor)

Joan Josep Ferrando •

Juan Antonio Sáez

\title{
Rainich theory for type D aligned Einstein-Maxwell solutions
}

Received: date / Revised version: date

\begin{abstract}
The original Rainich theory for the non-null Einstein-Maxwell solutions consists of a set of algebraic conditions and the Rainich (differential) equation. We show here that the subclass of type D aligned solutions can be characterized just by algebraic restrictions.
\end{abstract}

Keywords Rainich theory · Einstein-Maxwell · Type D

\section{Introduction}

The energy tensor $T$ associated with an electromagnetic field $F$ solution of the source-free Maxwell equations (Maxwell field), $\nabla \cdot F=0, d F=0$, is divergence-free, $\nabla \cdot T=0$. Conversely, if $T$ is a conserved symmetric tensor, what additional conditions must it satisfy in order to be the energy tensor of a Maxwell field? This problem was posed and solved by Rainich [1] for non-null fields obtaining, as a consequence, a fully geometric characterization of the non-null Einstein-Maxwell solutions. It is worth pointing out that the Rainich approach [1] also includes other interesting results about the principal planes of a non-null Maxwell field (see, for example 2] for a detailed analysis).

Here we are interested in the following points of the Rainich work: (i) to express Maxwell equations for the Maxwell-Minkowski energy tensor, such,

Send offprint requests to: Joan J. Ferrando

J.J. Ferrando

Departament d'Astronomia i Astrofísica,

Universitat de València, E-46100 Burjassot, València, Spain.

E-mail: joan.ferrando@uv.es

J.A. Sáez

Departament de Matemàtiques per a l'Economia i l'Empresa,

Universitat de València, E-46071 València, Spain.

E-mail: juan.a.saez@uv.es 
to obtain the algebraic conditions and the additional differential restrictions for a conserved symmetric tensor to be the energy tensor of a Maxwell field, and (ii) to write all these conditions, via Einstein equations, for the Ricci tensor considered as a metric concomitant.

The Rainich answer to the the first point can be summarized in the following [1]:

Theorem 1 (Rainich, 1925) A symmetric tensor $T$ is the energy tensor of a non-null Maxwell field if, and only if, it satisfies the algebraic conditions:

$$
\operatorname{tr} T=0, \quad 4 T^{2}=\operatorname{tr} T^{2} g \neq 0, \quad T(x, x)>0
$$

and the differential ones:

$$
\nabla \cdot T=0, \quad \mathrm{~d} \Psi[T]=0
$$

where $x$ is an arbitrary time-like vector and the Rainich 1 -form $\Psi[T]$ is given by

$$
\Psi[T] \equiv \frac{6}{\operatorname{tr} T^{2}} *(\nabla T \cdot T)
$$

The operators and products used in this theorem and hereafter are explicitly defined in Appendix A.

The answer to the second point of the Rainich work follows easily from the above theorem. Indeed, under the traceless condition, Einstein-Maxwell equations state that the Ricci tensor and the energy-momentum tensor differ in a constant. Thus, algebraic conditions (11) apply on the Ricci tensor. Moreover, field equations imply the conservation condition and only the Rainich (differential) equation must be imposed. Consequently, we have the following [1]:

Theorem 2 (Rainich, 1925) A metric tensor $g$ is a non-null EinsteinMaxwell solution if, and only if, its Ricci tensor $R \equiv R(g)$ satisfies the algebraic conditions:

$$
\operatorname{tr} R=0, \quad 4 R^{2}=\operatorname{tr} R^{2} g \neq 0, \quad R(x, x)>0
$$

and the differential one:

$$
\mathrm{d} \Psi(R)=0, \quad \Psi(R) \equiv \frac{6}{\operatorname{tr} R^{2}} *(\nabla R \cdot R)
$$

where $x$ is an arbitrary time-like vector.

Rainich-like characterizations have been considered for other matter models. Thus the Mariot-Robinson theorem [3,4] is the starting point in characterizing the null Einstein-Maxwell solutions. Several subsequent works have contributed to this goal (see, for example, Ref. [5,6]). In a different framework, in considering a hydrodynamical matter model, the local thermal equilibrium condition has been expressed in terms of energy tensor variables and a fully geometrical description of the thermodynamic perfect fluid solutions has been obtained [7]. 
It is worth remarking that in a Rainich-like approach we can limit the set of metrics where it applies or we can consider matter models with additional physical properties. Thus, the Rainich theory for the ideal gases in local thermal equilibrium has been useful in characterizing the ideal gas Stephani universes [8]. The conceptual and practical interest of these 'reduced' Rainich theories motivates the goal of this work: the characterization of the type $D$ aligned Einstein-Maxwell solutions.

This family of metrics contains significant solutions like the ReissnerNordström and the Kerr-Newman black holes and their vacuum limit, the Schwarzschild and Kerr solutions, as well as, other well-known space-times that generalize them, such as the charged Kerr-NUT solutions.

In posing this Rainich problem we look for the Einstein-Maxwell solutions in the set of the Petrov-Bel type D metrics whose principal 2-planes are those of the (non-null) Maxwell field. At first glance, we could solve this question by adding to the (algebraic and differential) Rainich conditions (4) and (5), the complementary algebraic conditions that state:

(i) The Weyl tensor is of Petrov-Bel type D.

(ii) The Weyl and Ricci tensors have aligned principal planes.

In this work we find explicit expressions in terms of the Ricci and Weyl tensors for the conditions (i) and (ii). But our results go quite a lot further. Indeed, we show that, under the alignment restriction (ii), the algebraic Rainich conditions (4) imply the type D requisite (i). On the other hand we show that, by adding a simple algebraic constraint, the algebraic conditions imply the (differential) Rainich equation (5). This means that no differential conditions are necessary to characterize the type D aligned Einstein-Maxwell solutions, that is, the two differential conditions (2), the divergence-free equation and the Rainich equation, follow from the field equations. More precisely, in this work we show the following:

Theorem 3 A metric tensor $g$ is a type D aligned Einstein-Maxwell solution if, and only if, its Ricci and Weyl tensors, $R \equiv R(g)$ and $W=W(g)$ satisfy the algebraic conditions:

$$
\begin{gathered}
\operatorname{tr} R=0, \quad 4 R^{2}=\operatorname{tr} R^{2} g \neq 0, \quad R(x, x)>0 \\
R_{(\alpha}^{\mu} P_{\beta) \mu \gamma \delta}=0, \quad A^{2}+B^{2} \neq 0, \quad B^{2}+\left(3 A-\operatorname{tr} R^{2}\right)^{2} \neq 0,
\end{gathered}
$$

where

$$
\begin{aligned}
& P \equiv W-\alpha G-\beta \eta, \quad \alpha \equiv-\frac{A C+B D}{A^{2}+B^{2}}, \quad \beta \equiv \frac{A D-B C}{A^{2}+B^{2}}, \\
& A \equiv \frac{1}{2} \operatorname{Tr} W^{2}, \quad B \equiv \frac{1}{2} \operatorname{Tr}(W \circ * W), \quad C \equiv \frac{1}{2} \operatorname{Tr} W^{3}, \quad D \equiv \frac{1}{2} \operatorname{Tr}\left(W^{2} \circ * W\right),
\end{aligned}
$$

and where $G=\frac{1}{2} g \wedge g$ is the metric on the 2 -forms space, $\eta$ is the metric volume element and $x$ is an arbitrary time-like vector.

The operators and products used in this theorem and hereafter are explicitly defined in Appendix A.

This work is organized as follows. In section 2 we give an alignment condition between the Weyl and Ricci tensors and show that, under this restriction, 
the algebraic Rainich conditions (4) imply that the Weyl tensor is Petrov-Bel type D. In section 3 we analyze the differential Rainich equation (5) and we show that it is identically satisfied by adding an algebraic scalar constraint to the algebraic restrictions studied in section 2, Moreover we justify the above theorem that characterizes the metric tensors $g$ and we give the procedure to obtain the Maxwell field $F$ which complete the Einstein-Maxwell solution. Section 4 is devoted to considering the counterpart solutions with cosmological constant and to analyze the vacuum limit. Finally, in order to clarify the notation used in the work, the operators and products are explicitly defined in Appendix A.

\section{Algebraic conditions}

Let $\left(V_{4}, g\right)$ be an oriented space-time of signature $\{-,+,+,+\}$, and let $R$ and $W$ be the Ricci and Weyl tensors, defined from the curvature tensor as given in [9].

A non-null electromagnetic field $F$ takes the canonical expression $F=$ $e^{\phi}[\cos \psi U+\sin \psi * U]$, where $U$ is a simple and unitary 2 -form that we name geometry of $F, \phi$ is the energetic index and $\psi$ is the Rainich index. The intrinsic geometry $U$ determines a $2+2$ almost-product structure defined by the time-like plane $V$ generated by the null eigenvectors of $F$ and whose volume element is $U$, and its space-like orthogonal complement $H$. They are the principal planes of $F$ (or of $U$ ). If $v$ and $h=g-v$ are the respective projectors and $\Pi=v-h$ is the structure tensor, we have $v=U^{2}$ and $h=-(* U)^{2}$, where $*$ denotes the Hodge dual operator and $U^{2}=U \cdot U$ (see Appendix A).

The (Maxwell-Minkowski) energy tensor $T$ associated with an electromagnetic field $F$ is minus the traceless part of its square and, in the non-null case, it depends on the intrinsic variables $(U, \phi)$ :

$$
T \equiv-\frac{1}{2}\left[F^{2}+* F^{2}\right]=-\frac{1}{2} e^{2 \phi}\left[U^{2}+* U^{2}\right]=-\kappa \Pi
$$

The symmetric tensor (8) has the principal planes of the electromagnetic field as eigenplanes and their associated eigenvalues are $\pm \kappa$, with $2 \kappa=$ $\sqrt{\operatorname{tr} T^{2}}=e^{2 \phi}$. Then, $T$ satisfies the conditions (11). Conversely, if a symmetric tensor satisfies the first two algebraic restrictions in (1), then it is, up to sign, the traceless part of the square of the simple 2 -form $F^{\circ}$ given by:

$$
F^{\circ}=F^{\circ}(T) \equiv \frac{Q(X)}{\sqrt{2 Q(X, X)}}, \quad Q \equiv T \wedge g-\frac{1}{\sqrt{\operatorname{tr} T^{2}}}(T \wedge g)^{2}
$$

where $X$ is an arbitrary 2 -form, $\wedge$ denotes the double-forms exterior product, and $S^{2}$ means the square of a double 2-form $S$ considered as an endomorphism on the 2-form space (see Appendix A).

But, in order to guarantee the physical meaning of an energy tensor $T$ we must also impose the energy conditions on it. Under the first two algebraic restrictions in (11) the Plebański energy conditions reduce to the third one. Consequently, (11) gives the algebraic characterization of a Maxwell-Minkowski 
energy tensor. Then, taking into account that the energy tensor determines the electromagnetic field up to duality rotation, we can state:

Lemma 1 A symmetric tensor $T$ is an energy tensor of non-null electromagnetic type if, and only if, it satisfies the algebraic conditions (11).

If $T$ satisfies (11) we can obtain the simple 2 -form $F^{\circ}$ given in (19). Then, for any scalar $\psi$, the electromagnetic field $F=\cos \psi F^{\circ}+\sin \psi * F^{\circ}$ has $T$ as its energy tensor.

In order to analyze the conditions for a space-time to be a Petrov-Bel type D solution we now introduce a necessary notation. A self-dual 2 -form is a complex 2 -form $\mathcal{F}$ such that $* \mathcal{F}=\mathrm{i} \mathcal{F}$. We can associate biunivocally with every real 2-form $F$ the self-dual 2 -form $\mathcal{F}=\frac{1}{\sqrt{2}}(F-\mathrm{i} * F)$. We here refer to a self-dual 2-form as a $S D$ bivector. The endowed metric on the 3 -dimensional complex space of the SD bivectors is $\mathcal{G}=\frac{1}{2}(G-\mathrm{i} \eta), \eta$ being the metric volume element of the space-time and $G$ the metric on the space of 2 -forms, $G=\frac{1}{2} g \wedge g$.

Every double-2-form, and in particular the Weyl tensor $W$, can be considered as an endomorphism on the space of the 2 -forms. The restriction of the Weyl tensor on the SD bivectors space is the self-dual Weyl tensor and is given by:

$$
\mathcal{W} \equiv \mathcal{G} \circ W \circ \mathcal{G}=\frac{1}{2}(W-\mathrm{i} * W)
$$

The algebraic classification of the Weyl tensor $W$ can be obtained by studying the traceless linear map defined by the self-dual Weyl tensor $\mathcal{W}$ on the SD bivectors space 10,11,12. The characteristic equation reads $x^{3}-$ $\frac{1}{2} a x-\frac{1}{3} b=0$, where the complex invariants $a$ and $b$ are given by:

$$
a \equiv \operatorname{Tr} \mathcal{W}^{2}, \quad b \equiv \operatorname{Tr} \mathcal{W}^{3} .
$$

In a Petrov-Bel type D space-time the self-dual Weyl tensor has a double eigenvalue and a minimal polynomial of degree two, and it admits the canonical expression [12]:

$$
\mathcal{W}=3 \rho \mathcal{U} \otimes \mathcal{U}+\rho \mathcal{G}, \quad \rho \equiv-\frac{b}{a}
$$

where $\mathcal{U}$ is the normalized eigenbivector associated with the simple eigenvalue $-2 \rho$. The canonical bivector $\mathcal{U}$ determines the $2+2$ structure $\Pi=2 \mathcal{U} \cdot \overline{\mathcal{U}}$, where ${ }^{-}$denotes the complex conjugate. The planes of the structure $\Pi$ are named the principal planes of a type D Weyl tensor. On the other hand, a type D Weyl tensor can be characterized as follows [12]:

Lemma 2 A space-time is of Petrov-Bel type D if, and only if, the self-dual Weyl tensor satisfies:

$$
a \neq 0, \quad \mathcal{W}^{2}-\frac{b}{a} \mathcal{W}-\frac{a}{3} \mathcal{G}=0
$$

where $a \equiv \operatorname{Tr} \mathcal{W}^{2}$ and $b \equiv \operatorname{Tr} \mathcal{W}^{3}$. 
In a type D aligned Einstein-Maxwell solution the energy tensor $T$ is of the electromagnetic type and its principal planes are those of the Weyl tensor. In this case the energy and Ricci tensors differ in a constant and, consequently, the Ricci tensor $R$ must satisfy the Rainich algebraic conditions (4).

In order to impose the alignment requirement, the following two conditions must hold: (i) $R=-\kappa \Pi, \Pi$ being the principal structure of the Weyl tensor, and (ii) The self dual Weyl tensor takes the form (11), $\mathcal{U}$ being the (self-dual) canonical unitary 2 -form of the electromagnetic field.

If we consider a frame of normalized SD bivectors $\left\{\mathcal{U}_{i}\right\}, \mathcal{U}_{1}=\mathcal{U}$, we have that the symmetric tensors $\left\{\mathcal{U}_{i} \cdot \overline{\mathcal{U}}_{j}\right\}$ define a (complex) basis of the space of the traceless symmetric tensors, and then the Ricci tensor may be written as $R=\sum R^{i j} \mathcal{U}_{i} \cdot \overline{\mathcal{U}}_{j}$. Moreover, any self-dual double 2 -form $P$ can be spanned as $P=\sum P^{i j} \mathcal{U}_{i} \otimes \mathcal{U}_{j}$. Then, some straightforward algebraic calculations enable the condition for the Ricci and Weyl tensors to have a given associated structure to be written as follows:

Lemma 3 (i) A space-time is algebraically of Einstein-Maxwell type with unitary principal bivector $\mathcal{U}$ if, and only if, the Ricci tensor $R$ satisfies:

$$
R \neq 0, \quad \operatorname{tr} R=0, \quad R \cdot \mathcal{U}=\mathcal{U} \cdot R
$$

(ii) A space-time is of Petrov-Bel type D with principal structure $\Pi$ if, and only if, the self-dual Weyl tensor $\mathcal{W}$ satisfies:

$$
a \neq 0, \quad \Pi_{(\alpha}^{\mu} \mathcal{P}_{\beta) \mu \gamma \delta}=0, \quad \mathcal{P} \equiv \mathcal{W}+\frac{b}{a} \mathcal{G}
$$

From this lemma we easily obtain the alignment condition in terms of the Ricci and Weyl tensors:

Lemma 4 A Ricci tensor $R$ of non-null electromagnetic type and a PetrovBel type $D$ Weyl tensor $W$ have aligned principal planes if, and, only if, they satisfy:

$$
a \neq 0, \quad R_{(\alpha}^{\mu} \mathcal{P}_{\beta) \mu \gamma \delta}=0, \quad \mathcal{P} \equiv \mathcal{W}+\frac{b}{a} \mathcal{G}
$$

where $\mathcal{W}=\frac{1}{2}(W-i * W)$ is the self-dual Weyl tensor and $a \equiv \operatorname{Tr} \mathcal{W}^{2}, b \equiv$ $\operatorname{Tr} \mathcal{W}^{3}$.

Until now we have shown that the algebraic restraints for a type D aligned Einstein-Maxwell solution are given by the Rainich conditions (4) on the Ricci tensor, the equations (12) characterizing a type D Weyl tensor and the alignment restriction (15). Nevertheless, all these conditions are excessive. Indeed, from lemma 3 a simple calculation shows that, under the alignment constraint (15), the second condition in (4) on the Ricci tensor is equivalent to restrictions (12) on the Weyl tensor. Thus we can state:

Proposition 1 The necessary and sufficient conditions for $g$ to be a type $D$ metric with principal planes aligned with a source tensor of electromagnetic type is that the Ricci tensor $R=R(g)$ and the self-dual Weyl tensor $\mathcal{W}=$ $\mathcal{W}(g)$ satisfy the algebraic restrictions (4) and (15).

Evidently, in the above proposition we can substitute the second condition in (4) by condition (12) that characterizes a type D Weyl tensor. 


\section{Differential conditions}

Once we have obtained the conditions that algebraically characterize the type D aligned Einstein-Maxwell solutions we will go on to study the differential constraints in this section. As pointed out in the introduction the conservation of the energy tensor, $\nabla \cdot T=0$, is a general consequence of the field equations. Consequently, a priory we must only impose the differential Rainich equation (5). Nevertheless, we will show now that this condition is unnecessary, that is, it is also a consequence of the field equations.

We begin this analysis bearing in mind the Maxwell-Rainich equations. Let $\Phi$ and $\Psi$ be the expansion vector and the rotation vector of the principal planes of an electromagnetic field, defined as [2,13]:

$$
\begin{aligned}
& \Phi \equiv \Phi[U] \equiv * U(\delta * U)-U(\delta U) \\
& \Psi \equiv \Psi[U] \equiv * U(\delta U)+U(\delta * U)
\end{aligned}
$$

Then, we have [1], 14:

Lemma 5 In terms of the intrinsic elements $(U, \phi, \psi)$ of a non-null Maxwell field, the source-free Maxwell equations, $\delta F=0, \delta * F=0$, take the expression:

$$
\mathrm{d} \phi=\Phi[U], \quad \mathrm{d} \psi=\Psi[U]
$$

When $F$ is solution of the source-free Maxwell equations, one says that $U$ defines a Maxwellian structure. Besides, when the Maxwell-Minkowski energy tensor $T$ associated with a non-null 2 -form is divergence-free, the underlying $2+2$ structure is said to be pre-Maxwellian [15]. The conservation of $T$ is equivalent to the first of the Maxwell-Rainich equations (17) 22. Then, from these equations we obtain the following result [1,2]:

Lemma 6 (i) A 2+2 structure is Maxwellian if, and only if, the expansion and the rotation are closed 1-forms, namely the canonical 2-form $U$ satisfies:

$$
\mathrm{d} \Phi[U]=0, \quad \mathrm{~d} \Psi[U]=0
$$

(ii) A $2+2$ structure is pre-Maxwellian if, and only if, the canonical 2form $U$ satisfies the first equation in (18).

This lemma enables us to obtain the differential constraints for the generic Rainich theory. Indeed, the algebraic conditions guarantee that a family of 2 -forms $F$ can be associated with the Ricci tensor (see lemma 1), and one of them must verify the Maxwell-Rainich equations (17). The first one is the conservation requirement, that is, a consequence of the field equation. The second one establishes that $\Psi$ is a closed 1-form, condition that gives the (differential) equation (5) if we write the rotation vector $\Psi$ in terms of the Ricci tensor [2].

In showing that this differential condition is unnecessary under the alignment requirement an important property of the type D aligned EinsteinMaxwell solution plays a central role: the two double Debever principal directions determine two shear-free geodesic null congruences (umbilical principal planes). Indeed, the type D aligned Einstein-Maxwell space-times with 
a non umbilical principal structure were studied by Plebański and Hacyan [17. They looked for solutions with cosmological constant and they found the 'exceptional' metrics, which are solutions with a non-vanishing cosmological constant. Thus, a type D aligned Einstein-Maxwell solution without cosmological constant has shear-free and geodesic null principal directions. We will show now that the umbilical condition is equivalent to a pair of algebraic constraints.

Let us consider an Einstein-Maxwell Ricci tensor, $R=-\kappa \Pi$, aligned with the type D Weyl tensor (11), $\Pi=2 \mathcal{U} \cdot \overline{\mathcal{U}}$. Then, the Bianchi equations take the expression:

$$
\begin{array}{r}
{\left[(3 \alpha-\kappa)^{2}+9 \beta^{2}\right] \tau_{v}=0, \quad\left[(3 \alpha+\kappa)^{2}+9 \beta^{2}\right] \tau_{h}=0} \\
\mathrm{~d} \kappa=2 \kappa \Phi \\
2 \mathrm{~d} \alpha=3(\alpha \Phi-\beta \Psi)+\kappa \Pi(\Phi), \quad 2 \mathrm{~d} \beta=3(\beta \Phi+\alpha \Psi)+\kappa \Pi(\Psi)
\end{array}
$$

where $\alpha$ and $\beta$ are the real and imaginary parts of the double Weyl eigenvalue, $\rho=\alpha+\mathrm{i} \beta, \Phi$ and $\Psi$ are the expansion and rotation vectors given in (16), and $\tau_{v}$ and $\tau_{h}$ are the traceless part of the symmetric second fundamental form of the principal planes (see, for example [13] for a specific definition). Considering $2 \kappa=e^{2 \phi}$, equation (20) becomes the first of the Maxwell-Rainich equations (17) and, consequently, it is precisely the conservation equation.

When $\tau_{v}$ and $\tau_{h}$ vanish the principal planes define an umbilical structure [13, and this geometric property is equivalent to the shear-free and geodesic character of the two null principal vectors [13, 16]. Thus, from the equations (19) we have that, if in a type D aligned Einstein-Maxwell space-time the Ricci eigenvalue $\kappa$ and the Weyl eigenvalue $\rho=\alpha+\mathrm{i} \beta$ satisfy

$$
(3 \alpha-\kappa)^{2}+9 \beta^{2} \neq 0, \quad(3 \alpha+\kappa)^{2}+9 \beta^{2} \neq 0,
$$

then the principal planes are umbilical, that is, the Debever principal directions define shear-free geodesic null congruences.

Now we show that the converse statement also holds: the solutions with umbilical principal planes satisfy, necessarily, the algebraic restrictions (22). Indeed, if the first (resp., second) condition in (22) does not hold, one has $\beta=0$ and $3 \alpha=\kappa$ (resp., $3 \alpha=-\kappa$ ). Then, Bianchi identities (20) and (21) imply that $\Phi=0$ and $v(\Psi)=0$ (resp., $\Phi=0$ and $h(\Psi)=0$ ). In [13] we have obtained the expression of the Ricci tensor in a space-time with an umbilical structure. In this case the Ricci tensor depends, up to two scalars, on the expansion and rotation vectors $\Phi$ and $\Psi$. When $\Phi=0$ the Ricci tensor becomes [13]:

$$
R=r_{v} v+r_{h} h+\Psi \otimes \Psi-* U \cdot \mathcal{L}_{\Psi} g \cdot U-U \cdot \mathcal{L}_{\Psi} g \cdot * U
$$

If one now imposes that $R=-\kappa \Pi=-\kappa(v-h)$, one obtains that $\Psi=0$. Thus, the principal planes are umbilical, minimal $(\Phi=0)$ and integrable $(\Psi=$ 0 ) and, consequently, $g$ becomes a product metric [13]. Now $R=r_{v} v+r_{h} h$ and the traceless condition implies $r_{v}+r_{h}=0$. But, for a product metric, this condition leads to a vanishing Weyl tensor, that contradicts our type D 
hypothesi. Consequently, under the umbilical property, (22) applies and we can state:

Lemma 7 In a type $D$ aligned Einstein-Maxwell space-time the Debever principal directions define shear-free geodesic null congruences if, and only if, the Ricci eigenvalue $\kappa$ and the Weyl eigenvalue $\rho=\alpha+i \beta$ satisfy (22).

Elsewhere [13] we have studied the integrability conditions for the umbilical condition and we have obtained interesting consequences for the different Petrov-Bel types. A detailed analysis of the type D space times leads to the following (see proposition 10 in reference [13]):

Lemma 8 In a type D space-time whose Debever null principal directions are shear-free and geodesic (the principal planes are umbilical), if the principal structure is pre-Maxwellian, $\mathrm{d} \Phi=0$, then it is Maxwellian, $\mathrm{d} \Phi=\mathrm{d} \Psi=0$.

This lemma implies that, under the umbilical condition, the differential Rainich equation (5) is a consequence of the field equations. But lemma 7 states that the umbilical (differential) condition can be expressed, equivalently, by means of the (algebraic) constraint (22). On the other hand, remembering that, as a consequence of the work by Plebański and Hacyan [17, all the type D aligned Einstein-Maxwell solutions without cosmological constant have umbilical principal planes, and taking into account proposition 1, we can state:

Proposition 2 The necessary and sufficient conditions for $g$ to be a type $D$ aligned Einstein-Maxwell solution is that the Ricci tensor $R=R(g)$ and the self-dual Weyl tensor $\mathcal{W}=\mathcal{W}(g)$ satisfy the algebraic restrictions (4), (15) and (22).

We can make the characterization given in the above proposition more explicit with the following considerations. From the expression (11) we obtain the real imaginary part of the Weyl eigenvalue as

$$
\alpha \equiv-\frac{A C+B D}{A^{2}+B^{2}}, \quad \beta \equiv \frac{A D-B C}{A^{2}+B^{2}},
$$

where $A, B, C, D$ are the real invariants of the Weyl tensor that are related to the complex ones by $2 a=A-\mathrm{i} B, 2 b=C-\mathrm{i} D$.

On the other hand, the alignment equation (15) is equivalent to its real part since the Ricci tensor $R$ is real and $\mathcal{P}$ is a self-dual double $2-$ form. Thus, taking the real part in (15) we can write this condition in terms of the (real) Weyl tensor and its real invariants. Finally, the two constraints (22) state, equivalently, that $6 a \neq \operatorname{tr} R^{2}$. Taking into account all these considerations proposition 2 implies our main theorem 3 settled in the introduction.

Theorem 3 characterizes the family of type D aligned Einstein-Maxwell space-times by means of algebraic conditions. In order to complete the Einstein-Maxwell solution we need to obtain the Maxwell field $F$ associated with the Maxwell-Minkowski energy tensor $T$. If we take into account the MaxwellRainich equations (17), the expression $\Psi(R)$ in (5) of the rotation vector in terms of the Ricci tensor, and the second statement in lemma 1, we arrive to the following result: 
Proposition 3 Let $g$ be a metric tensor satisfying the conditions (6) and (7) of theorem 3. Then, a function $\psi$ exists such that $\mathrm{d} \psi=\Psi(R)$ where $\Psi(R)$ is given in (5). Let us consider the 2 -form $F=\cos \psi F^{\circ}+\sin \psi * F^{\circ}$, where $F^{\circ}=F^{\circ}(R)$ is given in (9). Then the pair $(g, F)$ is an Einstein-Maxwell solution.

\section{On the solutions with cosmological constant and on the vacuum limit}

The explicit integration of the Einstein-Maxwell equations for the family of metrics characterized in theorem 3 has been obtained by several authors (see 18, 19, 20, and references therein). This family of solutions, also including their counterpart with cosmological constant, has been named the $\mathcal{D}$-metrics 20. They can be deduced from the Plebańsky and Demiański 18 line element by means of several limiting procedures (see 9] and references therein and 21] for a recent analysis).

The Rainich-like characterization of the $\mathcal{D}$-metrics easily follows from the analysis presented in the previous sections. Indeed, the algebraic conditions applies if we replace the Ricci tensor by its traceless part and impose a constant trace. On the other hand, in showing that the differential Rainich condition is unnecessary we have used the Bianchi equations (19/20|21) that take exactly the same expression when the cosmological constant is present. Thus, we can state:

Theorem 4 A metric tensor $g$ is a charged $\mathcal{D}$-metric if, and only if, its Ricci and Weyl tensors, $R \equiv R(g)$ and $W=W(g)$ satisfy the conditions:

$$
\begin{array}{r}
\mathrm{d} \operatorname{tr} R=0, \quad 4 \tilde{R}^{2}=\operatorname{tr} \tilde{R}^{2} g \neq 0, \quad \tilde{R}(x, x)>0 \\
R_{(\alpha}^{\mu} P_{\beta) \mu \gamma \delta}=0, \quad A^{2}+B^{2} \neq 0, \quad B^{2}+\left(3 A-\operatorname{tr} \tilde{R}^{2}\right)^{2} \neq 0
\end{array}
$$

where $P, A$, and $B$ depend on the Weyl tensor as given in theorem $3, \tilde{R}$ is the traceless part of the Ricci tensor, $\tilde{R} \equiv R-\frac{1}{4} \operatorname{tr} R g$, and where $x$ is an arbitrary time-like vector.

It is worth remarking that theorem 4 does not give the Rainich-like characterization of the whole set of type D aligned Einstein-Maxwell solutions with cosmological constant. Indeed, theorem 4 characterizes the $\mathcal{D}$-metrics, and this family does not contain the exceptional metrics by Plebański and Hacyan [17.

Let us also note that the space-times which theorems 3 and 4 characterize do not include the vacuum limit. Indeed, the algebraic Rainich conditions (24) avoid the vacuum case $R=0$ and, moreover, the alignment condition (first in (25) ) holds identically and does not guarantee a Weyl tensor of type D.

In order to obtain a Rainich-like characterization of the $\mathcal{D}$-metrics that also includes the vacuum solutions we must replace the second condition in (24) by the conditions (12) for a type D Weyl tensor. Then, we obtain an alternative characterization that we give in complex vectorial formalism: 
Theorem 5 A metric tensor $g$ is a $\mathcal{D}$-metric (including the vacuum limit) if, and only if, its Ricci and Weyl tensors, $R \equiv R(g)$ and $W=W(g)$ satisfy the conditions:

$$
\begin{array}{cc}
\mathrm{d} \operatorname{tr} R=0, & \tilde{R}(x, x) \geq 0 \\
a \neq 0, \quad & \mathcal{W}^{2}-\frac{b}{a} \mathcal{W}-\frac{a}{3} \mathcal{G}=0 \\
R_{(\alpha}^{\mu} \mathcal{P}_{\beta) \mu \gamma \delta}=0, & 6 a \neq \operatorname{tr} \tilde{R}^{2}
\end{array}
$$

where

$$
\begin{aligned}
\tilde{R} & \equiv R-\frac{1}{4} \operatorname{tr} R g, & \mathcal{P} \equiv \mathcal{W}+\frac{b}{a} \mathcal{G}, \quad a \equiv \operatorname{Tr} \mathcal{W}^{2}, \quad b \equiv \operatorname{Tr} \mathcal{W}^{3}, \\
\mathcal{G} \equiv \frac{1}{2}(G-i \eta), & \mathcal{W} \equiv \frac{1}{2}(W-i * W), &
\end{aligned}
$$

and where $G=\frac{1}{2} g \wedge g$ is the metric on the 2 -forms space, $\eta$ is the metric volume element and $x$ is an arbitrary time-like vector.

The main results of this work (theorems 3, 4 and 5) offer an intrinsic (depending solely on the metric tensor) and explicit characterization of an important family of known solutions of the Einstein equations: the $\mathcal{D}$-metrics. This kind of characterization has already been given for two subsets of this family: the static type D vacuum solutions [22] and their charged counterpart 16. The results in these quoted works have allowed us to obtain an intrinsic and explicit labeling of every metric of these families and, in particular, to characterize the Schwarzschild and Reissner-Nordström black holes [22 [16].

In a similar way, the characterization of the $\mathcal{D}$-metrics that we give in the present work is a first step in labeling every particular solution in this family. This task and, in particular, the intrinsic characterization of the Kerr and Kerr-Newman black holes will be tackled elsewhere [23].

Acknowledgements This work has been partially supported by the Spanish Ministerio de Educación y Ciencia, MEC-FEDER project FIS200606062 .

\section{A Notation}

1. Products and other formulas involving 2-tensors $A$ and $B$ :

(a) Composition as endomorphisms: $A \cdot B$,

$$
(A \cdot B)_{\beta}^{\alpha}=A_{\mu}^{\alpha} B_{\beta}^{\mu}
$$

(b) Square and trace as an endomorphism:

$$
A^{2}=A \cdot A, \quad \operatorname{tr} A=A_{\alpha}^{\alpha} .
$$

(c) Action on a vector $x$, as an endomorphism $A(x)$, and as a quadratic form $A(x, x)$ :

$$
A(x)^{\alpha}=A_{\beta}^{\alpha} x^{\beta}, \quad A(x, x)=A_{\alpha \beta} x^{\alpha} x^{\beta} .
$$

(d) Exterior product as double 1-forms: $(A \wedge B)$,

$$
(A \wedge B)_{\alpha \beta \mu \nu}=A_{\alpha \mu} B_{\beta \nu}+A_{\beta \nu} B_{\alpha \mu}-A_{\alpha \nu} B_{\beta \mu}-A_{\beta \mu} B_{\alpha \nu} .
$$


2. Products and other formulas involving double 2-forms $P$ and $Q$ :

(a) Composition as endomorphisms of the bivectors space: $P \circ Q$,

$$
(P \circ Q)_{\rho \sigma}^{\alpha \beta}=\frac{1}{2} P_{\mu \nu}^{\alpha \beta} Q_{\rho \sigma}^{\mu \nu}
$$

(b) Square and trace as an endomorphism:

$$
P^{2}=P \circ P, \quad \operatorname{Tr} P=\frac{1}{2} P_{\alpha \beta}^{\alpha \beta} .
$$

(c) Action on a bivector $X$, as an endomorphism $P(X)$, and as a quadratic form $P(X, X)$,

$$
P(X)^{\alpha \beta}=\frac{1}{2} P_{\mu \nu}^{\alpha \beta} X^{\mu \nu}, \quad P(X, X)=\frac{1}{4} P_{\alpha \beta \mu \nu} X^{\alpha \beta} X^{\mu \nu} .
$$

3. Other products and operators:

(a) The Hodge dual operator is defined as the action of the metric volume element $\eta$ on a bivector $F$ and a double 2 -form $W$ :

$$
* F=\eta(F), \quad * W=\eta \circ W .
$$

(b) For a tensor $t, *(t)$ denotes the action of the Hodge dual operator on the skew-symmetric part of $t$. For example,

$$
*(\nabla T \cdot T)_{\alpha}=\frac{1}{6} \eta_{\alpha}^{\beta \mu \nu} \nabla_{\beta} T_{\mu \lambda} T_{\nu}^{\lambda} .
$$

Note that the symbol - indicates, as in the endomorphism composition, the contraction of the adjacent indices of the tensorial product.

\section{References}

1. Rainich, G.Y.: Trans. Am. Math. Soc. 27, 106 (1925)

2. Ferrando, J.J., Sáez, J.A.: Gen. Relativ. Gravit. 35, 1191 (2003)

3. Mariot, L.: C. R. Acad. Sc, Paris 2382055 (1954)

4. Robinson, I.: J. Math. Phys. 2290 (1961)

5. Bartrum, P.C.: J. Math. Phys. 8667 (1967)

6. Ludwig, G.: Commun. math. Phys. 1798 (1970)

7. Coll, B., Ferrando, J.J.: J. Math. Phys. 30, 2918 (1989)

8. Coll, B., Ferrando, J.J.: Gen. Rel. Grav. 37, 557 (2005)

9. Stephani, H., Kramer, D., MacCallum, M., Hoenselaers, C., Herlt, E.: Exact solutions to Einstein's field equations (Cambridge University Press, Cambridge, 2003)

10. Petrov, A.Z.: Sci. Not. Kazan Univ. 114, 55 (1954). This article has been reprinted in Gen. Rel. Grav. 32, 1665 (2000)

11. Bel, L.: Cah. de Phys. 16 59, (1962). This article has been reprinted in Gen. Rel. Grav. 32, 2047 (2000)

12. Ferrando, J.J., Morales, J.A., Sáez, J.A.: Class. Quantum Grav. 18, 4969 (2001)

13. Ferrando, J.J., Sáez, J.A.: Gen. Relativ. Gravit. 39, 343 (2007)

14. Coll, B., Fayos, F., Ferrando, J.J.: J. Math. Phys. 28, 1075 (1987)

15. Debever, R.: Bull. Acad. R. Belgique 62, 662 (1976)

16. Ferrando, J.J., Sáez, J.A.: J. Math. Phys. 45, 652 (2004)

17. Plebański, J.F, Hacyan, S.: J. Math. Phys. 20, 1004 (1979)

18. Plebański, J.F., Demiański, M.: Ann. Phys. (NY) 9898 (1976)

19. Weir, G.J., Kerr, R.P.: Proc. Roy. Soc, A, 35531 (1977)

20. Debever, R., Kamran, N., McLenaghan, R.C.: J. Math. Phys. 25, 1955 (1984)

21. Griffiths, J.B., Podolský J.: Int. J. Mod. Phys. D 15, 335 (2006)

22. Ferrando, J.J., Sáez, J.A.: Class. Quantum Grav. 15, 1323 (1998)

23. Ferrando, J.J., Sáez, J.A.: (in preparation) 